\title{
Annexin A1, A2, A4 and A5 play important roles in breast cancer, pancreatic cancer and laryngeal carcinoma, alone and/or synergistically
}

\author{
SHISHAN DENG ${ }^{1,2}$, JIANGUO WANG $^{1}$, LINGMI HOU ${ }^{3}$, JINSUI LI $^{3}$, GUO CHEN $^{4}$, \\ BAOQIAN JING ${ }^{4}$, XIAOMING ZHANG ${ }^{5}$ and ZHENGWEI YANG ${ }^{2}$ \\ ${ }^{1}$ Department of Anatomy and ${ }^{2}$ Morphometric Research Laboratory, North Sichuan Medical College; \\ ${ }^{3}$ Department of General Surgery, The Affiliated Hospital of North Sichuan Medical College; \\ ${ }^{4}$ Institute of Immunology and Molecular Biology, and ${ }^{5}$ Sichuan Key Laboratory of Medical Imaging, \\ North Sichuan Medical College, Nanchong, P.R. China
}

Received May 28, 2012; Accepted August 22, 2012

DOI: $10.3892 / \mathrm{ol} .2012 .959$

\begin{abstract}
Annexins are associated with metastasis and infiltration of cancer cells. Proteomic analysis and immunohistochemical staining were used to understand whether several annexins play important roles in cancer alone and/ or synergistically. Seven fresh breast cancer samples with 23 paraffin specimens, three fresh pancreatic samples and five fresh laryngeal carcinoma samples with 25 paraffin specimens were obtained from humans, as well as ten golden hamster pancreatic cancer tissue samples, and they were used to observe differential expression of annexins compared with normal tissues using proteomics and immunohistochemical staining. Annexin A2, A4 and A5 were overexpressed in human breast cancer and laryngeal carcinoma tissues and in golden hamster pancreatic cancer tissue samples, respectively, as shown by proteomics and immunohistochemical staining. In addition, annexin A4 and A5 were expressed in breast cancer tissues, while annexin A1 was not expressed. Annexin A1, A2 and A4 were expressed in human laryngeal carcinoma tissues as shown by immunohistochemical staining. Annexin A1, A2, A4 and A5 played important roles in breast cancer, pancreatic cancer and laryngeal carcinoma, alone and/or synergistically, and they may be targets of therapy for malignant tumors. The choice of which annexins to target should depend on their respective biological behaviors.
\end{abstract}

Correspondence to: Dr Shishan Deng, Department of Anatomy, North Sichuan Medical College, 234 Fujiang Road, Nanchong 637007, P.R. China

E-mail: dssgeneral@163.com

Key words: breast cancer, pancreatic cancer, laryngeal carcinoma, annexins, proteomics, immunohistochemical staining, overexpression

\section{Introduction}

The annexin family of calcium-dependent phospholipid binding proteins, as molecules that promote cancer cell invasion and metastases, play important roles in certain types of cancer, such as breast cancer, gastric cancer, esophageal carcinoma, liver cancer and nasopharyngeal carcinoma (1-5). In certain cancer tissue, some annexins are upregulated but others are downregulated. Therefore, these annexins, including upregulated and downregulated members, synergistically play important roles in cancer $(6,7)$. At present, studies have mainly focused on the role of single members of the annexin family in each type of cancer, but the synergistic roles that are played by several annexins are usually ignored. It is unclear whether a single annexin may play a major role in a type of cancer and other annexins minor roles.

In the physiological activity of the cells, an abundance of protein is usually associated with the strength of its effect. The different annexins may play diverse roles, so we observed them from several different common malignant tumors using differential proteomics analysis and immunohistochemical techniques to research their respective effects.

\section{Materials and methods}

Human tissue samples. Seven samples of fresh breast cancer and adjacent normal tissues (three samples from stage T1N1M0, three from T1N2M0 and one from T2N2M0) were obtained after surgery (mastectomy) in female patients aged from 35 to 65 years suffering from breast cancer, who were hospitalized in the Affiliated Hospital of North Sichuan Medical College. A total of 23 paraffin specimens of human breast infiltrating ductal carcinoma were obtained from the Department of Pathology in this hospital.

Three samples of fresh pancreatic cancer and adjacent normal tissues (two samples from T1N2M0 stage and one from T1N3M0) were obtained following surgery in male patients aged from 35 to 50 years suffering from pancreatic 
cancer, who were hospitalized in the Affiliated Hospital of North Sichuan Medical College.

Five groups of fresh laryngeal carcinoma and adjacent normal tissues (one sample from stage T1N1M0, two from $\mathrm{T} 1 \mathrm{~N} 2 \mathrm{M} 0$, one from T1N3M0 and one from T2N2M0) were obtained following surgery (laryngectomy) in male patients aged from 45 to 60 years suffering from laryngeal carcinoma, who were hospitalized in the Affiliated Hospital of North Sichuan Medical College. A total of 25 paraffin specimens of human laryngeal squamous carcinoma were obtained from the Department of Pathology in this hospital.

These three groups of fresh samples were immediately frozen at $-150^{\circ} \mathrm{C}$ (Super Low Temperature Icebox, Revco, CT, USA). Care was taken to ensure that the samples were from cancer tissues with non-necrotic, non-purulent and nonhemorrhagic characteristics. Breast cancer, pancreatic cancer and laryngeal carcinoma were confirmed as infiltrating ductal carcinoma, duct adenocarcinoma and squamous carcinoma, respectively, by pathological analysis.

The research was approved by the Ethics Committee of North Sichuan Medical College and all patients voluntarily provided written informed consent to participate in the study.

Golden hamster pancreatic cancer tissue samples. In total, 127 female golden hamsters from 7 to 8 weeks of age and from 250 to $300 \mathrm{~g}$ in weight were purchased from the experimental animal center of North Sichuan Medical College and divided into two groups: the experimental group included 100 golden hamsters and the control group included 27 golden hamsters. The experimental group was administered BOP [N-nitroso-bis(2-oxo-propyl)amine] (C6H10N2O3, International Laboratory, San Francisco, CA, USA), which was dissolved in saline $(1 \mathrm{mg} / \mathrm{ml})$. BOP $(10 \mathrm{mg} / \mathrm{kg})$ was subcutaneously injected into the experimental group, but the control group was only injected with $1 \mathrm{ml}$ saline. BOP was used once a week for a total of seven weeks and then 20 golden hamsters of the experimental group were randomly selected to undergo rosiglitazone gavage with $0.01 \mathrm{mg} / \mathrm{ml}$ and $10 \mathrm{ml} / \mathrm{kg}$ once a day for a total of five months. Finally, ten pancreatic cancer models with more than $50 \mathrm{mg}$ of cancer tissue from each golden hamster were successfully obtained, and the tumors were identified as pancreatic adenocarcinoma by pathological analysis.

The experiment was performed on the condition that the use of golden hamsters followed internationally recognized guidelines on animal welfare, as well as Nanchong's and Chinese regulations.

Protein extraction. Total proteins from fresh tissues were respectively obtained with a ReadyPrep ${ }^{\mathrm{TM}}$ Protein Extraction kit (GE, Fairfield, CT, USA) and then were stored in aliquots of $200 \mu \mathrm{l}$ at $-80^{\circ} \mathrm{C}$. Concentrations of total proteins were measured with Bio-Rad RC DC Protein Assay (GE). As some substances were contained in total proteins, such as salts, nucleic acids and lipids, which may interfere with isoelectronic focusing (IEF), ReadyPrep $^{\text {TM }}$ Cleanup kits (GE) were used to wash them away.

Proteomics analysis. Protein samples prepared in advance were separated by two-dimensional polyacrylamide gel electrophoresis (2-DE). Isoelectronic focusing (IEF) was performed with precast IPG strips (pH 3.0-10.0, nonlinear pH IPG, $17 \mathrm{~cm}, \mathrm{GE}$ ) using the focusing tray (GE). For IEF, the loading volume of the protein sample was $300 \mu \mathrm{l}$ while the loading content was $300 \mu \mathrm{g}$. IEF was carried out at $20^{\circ} \mathrm{C}$ and $50 \mu \mathrm{A} /$ strip. Following IEF, the strips were equilibrated, and then placed on top of the $12 \%$ SDS polyacrylamide gels prepared and sealed in place with $0.5 \%$ low-melt agarose (Sigma, St. Louis, MO, USA). The sodium dodecyl sulphate-polyacrylamide gel electrophoresis (SDS-PAGE) was performed at a current of $16 \mathrm{~mA} / \mathrm{gel}$ for $30 \mathrm{~min}$ and then at another current of $24 \mathrm{~mA} / \mathrm{gel}$ at $4^{\circ} \mathrm{C}$ for approximately $5 \mathrm{~h}$ using PROTEAN II ${ }^{\circledR}$ Xi Cell (Bio-Rad, Hercules, CA, USA). After SDS-PAGE, each gel was stained with coomassie brilliant blue R-250 (Amresco, NY, USA) and scanned with UMAXpowerlook 1120 (UMAX, Taipei, Taiwan), and then analyzed using PDQuest software (7.1) and Microsoft Office Excel 2003 to obtain differential protein spots and analyze reproducibility. Student's t-test was used for the statistical analysis of differential proteins.

Differential protein spots from gels were identified with matrix-assisted laser desorption/ionization tandem time-offlight mass tandem spectrometry (MALDI-TOF-TOF MS/MS), which was performed on an ABI 4700 TOF-TOF Proteomics Analyzer (Applied Biosystems, Foster, CA, USA). The UV laser was operated at a $200 \mathrm{~Hz}$ repetition rate with a wavelength of $355 \mathrm{~nm}$, and the accelerated voltage was operated at $20 \mathrm{kV}$, and mass resolution was maximized at $1500 \mathrm{Da}$. Myoglobin digested with trypsin was used to calibrate the mass spectrometry instrument using the internal calibration mode, and all spectra of samples acquired were processed using 4700 Explore $^{\mathrm{TM}}$ Software (Applied Biosystems) in a default mode. Finally, the data were searched by GPS Explorer (V3.6) with the search engine MASCOT 2.1, and the search parameters were as follows: the database NCBInr (NCBI2009: 9874297 sequences, 3367796496 residues), taxonomy Homo sapiens [human], protein molecular mass ranged from 700 to 3,000 Da, trypsin digest with one missing cleavage, MS tolerance was set at 0.3 Da, and MS/MS tolerance at 0.4 Da. Proteins with scores $>59$ or best ion scores $(\mathrm{MS} / \mathrm{MS})>30$ were significant $(\mathrm{P}<0.05)(8)$.

Immunohistochemical staining. Besides ready paraffin samples obtained from the Department of Pathology, fresh iced tissue samples were cut into small blocks (4-6 mm in length) and fixed in $10 \%$ formalin solution. After dehydration in ethanol and clearing in xylene, tissue blocks were embedded in paraffin and sectioned at $5 \mu \mathrm{m}$ in thickness. Immunohistochemical staining was performed in three different cancer tissues including breast cancer, pancreatic cancer and laryngeal carcinoma. Ultrasensitive ${ }^{\mathrm{TM}}$ SP kits (Maixin-Bio, Fuzhou, China) were used with anti-annexin A1 antibody (Boster, Wuhan, China), anti-annexin A2 antibody (Abcam, Cambridge, UK), anti-annexin A4 antibody (BOSTER, Wuhan, China) and anti-annexin A5 antibody (Boster), respectively, as the primary antibody (dilution 1:50). The results were statistically analyzed with SPSS1 (3.0) and $\chi^{2}$ test.

\section{Results}

Using PDQuest software (7.1) and Microsoft Office Excel 2003, positional deviation of the protein spot was $1.458 \pm 0.234 \mathrm{~mm}$ 
Table I. Three proteins were identified as annexin A2, A4 and A5, respectively, by MALDI-TOF-TOF MS/MS analysis.

\begin{tabular}{|c|c|c|c|c|c|c|c|}
\hline $\begin{array}{l}\text { Serial } \\
\text { No. }\end{array}$ & $\begin{array}{l}\text { Protein } \\
\text { Name }\end{array}$ & $\begin{array}{l}\text { Protein } \\
\text { MW (Da) }\end{array}$ & $\begin{array}{l}\text { Protein } \\
\text { PI }\end{array}$ & $\begin{array}{l}\text { Pep. } \\
\text { count }\end{array}$ & $\begin{array}{c}\text { Accession } \\
\text { No. }\end{array}$ & Tumor type & Tissue origin \\
\hline 1 & Annexin A2 & 40502.8 & 8.41 & 13 & gil73909156 & Breast cancer & Human \\
\hline 2 & Annexin A4 & 35871.1 & 5.42 & 16 & gil55742832 & Pancreatic cancer & Golden hamster \\
\hline 3 & Annexin A5 & 35783.4 & 4.94 & 28 & gil809185 & Laryngeal carcinoma & Human \\
\hline
\end{tabular}

Table II. Positive samples of annexin A1, A2, A4 and A5 in two cancer tissues were identified by immunohistochemical staining and statistical analysis from a total of thirty samples $(\mathrm{P}<0.05)$.

\begin{tabular}{lcccc}
\hline Origins of tissues & Annexin A1 (\%) & Annexin A2 (\%) & Annexin A4 (\%) & Annexin A5 (\%) \\
\hline Breast cancer tissues & $6(20.0)$ & $25(83.3)$ & $21(70.0)$ & $23(76.7)$ \\
Laryngeal carcinoma tissues & $20(66.7)$ & $24(80.0)$ & $22(73.3)$ & $24(80.0)$ \\
\hline
\end{tabular}

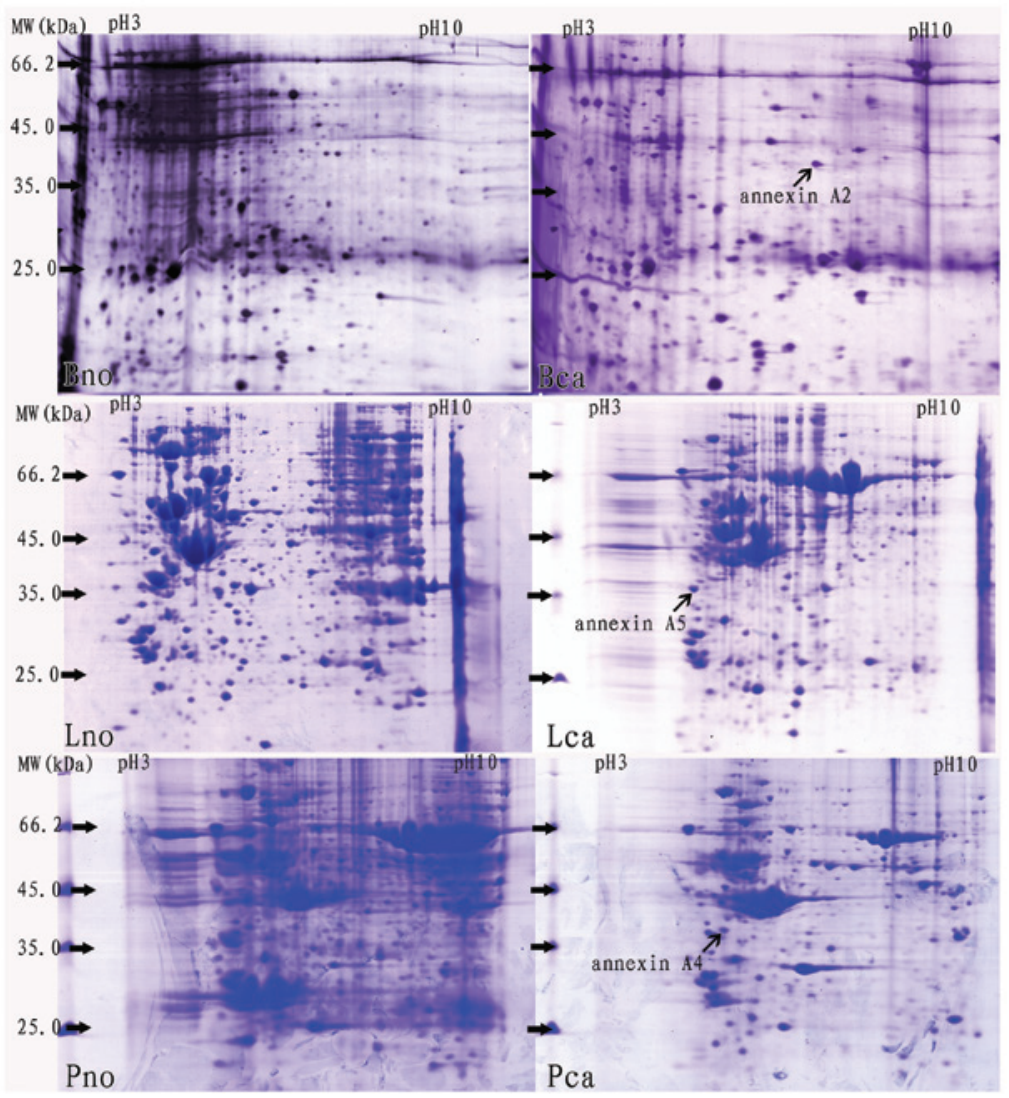

Figure 1 Three differential protein spots from 2-DE gels were identified as annexin A2, A4 and A5 $(\rightarrow)$, respectively, by differential proteomics analysis, and the proteins were individually overexpressed in human breast cancer tissues (Bca), human laryngeal carcinoma tissues (Lca) and golden hamster pancreatic cancer tissues (Pca). 2-DE, two-dimensional polyacrylamide gel electrophoresis. Lno, normal laryngeal mucosal tissues; Pno, normal pancreatic tissues; Bno, normal breast tissues.

for IEF and 1.012 $\pm 0.178 \mathrm{~mm}$ for 2-DE. Following 2-DE, differential protein spots were analyzed with PDQuest software (7.1) ( $>2$ folds), and then they were identified by MALDI-TOF-TOF MS/MS analysis. Among the differential proteins identified, certain annexins were overexpressed in various cancer tissues, including annexin A2, A4 and A5, respectively (Fig. 1 and Table I).
Furthermore, immunohistochemical staining analysis was performed to identify expression of these three annexins in the three corresponding groups of tissues, and the results show that annexin A2 (83.3\%) was expressed in thirty human breast cancer tissue samples at a higher level than normal, similarly, annexin A4 (100\%) was overexpressed in ten golden hamster pancreatic cancer tissue samples and annexin A5 (80.0\%) was 


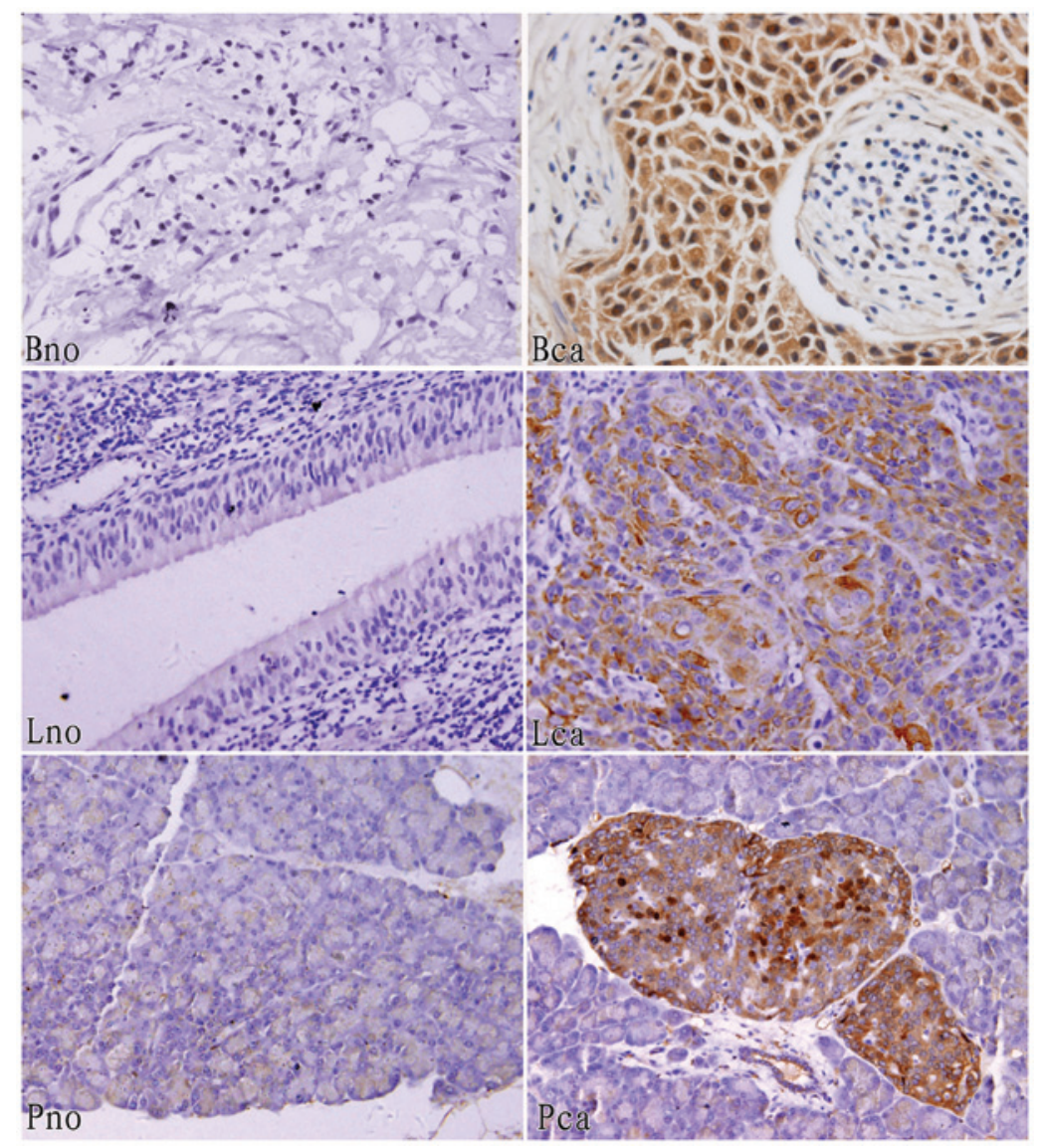

Figure 2. Three annexins that were further identified as positively expressed by immunohistochemical staining analysis were overexpressed in human breast cancer tissues (Bca), human laryngeal carcinom tissues (Lca) and golden hamster pancreatic cancer tissues (Pca), respectively.

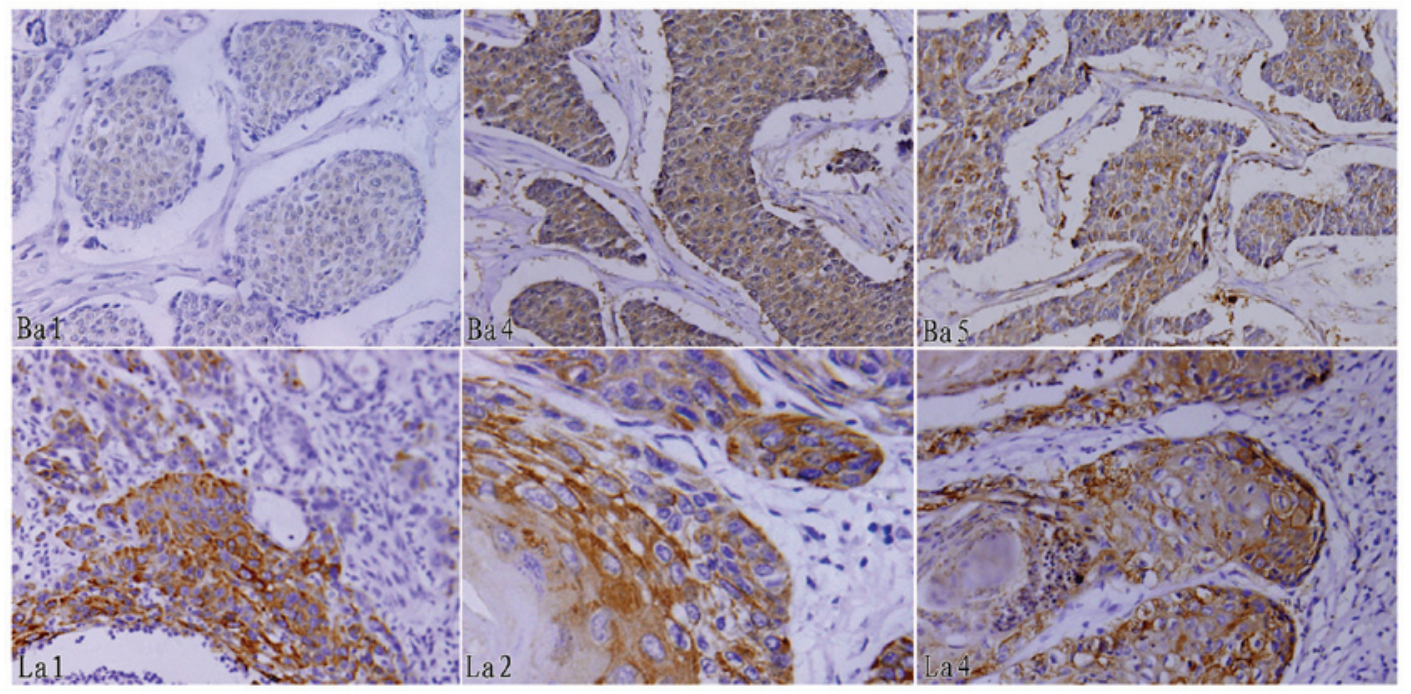

Figure 3. By immunohistochemical staining analysis, annexin A4 (Ba4) and annexin A5 (Ba5) were shown to be positively expressed in breast cancer tissues but annexin A1 (Ba1) was negatively expressed. Annexin A1 (La1), annexin A2 (La2) and annexin A4 (La4) were expressed positively in human laryngeal carcinoma tissues.

overexpressed in thirty human laryngeal carcinoma tissue samples (Fig. 2 and Table II).

Using human pancreatic cancer samples and adjacent normal tissues, we observed no annexins that were expressed differentially by differential proteomic analysis; however, annexin A4 was expressed in pancreatic cancer tissues at a higher level than normal in golden hamsters. By immunohistochemical staining analysis, we found that annexin A4 (70.0\%) and A5 (76.7\%) were expressed positively in breast cancer tissues but annexin A1 (80.0\%) was negatively expressed, while annexin A1 
(66.7\%), A2 (80.0\%) and A4 (73.3\%) were positively expressed in human laryngeal carcinoma tissues (Fig. 3 and Table II).

\section{Discussion}

In the very early stages of cancer, invasion and metastases of cancer cells often have occurred in patients prior to typical symptoms are found, and invasion and metastases of cancer cells often result in treatment failure. It was reported that invasion and metastases of cancer cells were associated with many factors, such as tumor metastasis associated gene 1 (MTA-1), matrix metalloproteinase inhibitors (TIMPs), hypoxia inducible factor $1 \alpha(\mathrm{HIF}-1 \alpha), \mathrm{nm} 23-\mathrm{H} 1$ and annexins (9-12). Among these factors, annexins may be linked with certain signaling pathways, including cell growth, transformation and apoptosis pathways. The majority of annexins contribute to invasion and metastases of cancer cells, and every member mainly affected a type of cancer cell. Annexin A1 is related to esophageal cancer, while annexin A2 is associated with breast cancer $(13,14)$. However, invasion and metastases of cancer cells are complicated biological activities and involve many important functional proteins; therefore, it is impossible that only one protein is responsible for such activities. The results indicated that proteins may synergistically affect the progression of malignant tumors.

By differential proteomics analysis, we identified that annexin A2, A4 and A5 were upregulated in breast cancer, pancreatic cancer and laryngeal carcinoma tissues, respectively, and the results indicated that a specific type of cancer cell could be affected by a specific annexin that plays a main role in a type of malignant tumor. Attention has now been drawn to whether they also play important roles in other types of cancer. According to previous studies, annexin A2 was also related to prostate cancer, invasive cervical carcinoma and lung cancer (15-17,18); annexin A4 was involved in gastric cancer and colorectal cancer (2,19); annexin A5 was associated with nasopharyngeal carcinoma and colorectal adenocarcinomas. The results indicated that other proteins, apart from the three annexins, may synergistically affect the progress of the three types of cancer cells. We also observed that annexin A4 and A5 were highly expressed in breast cancer tissues, while annexin A1, A2 and A4 in human laryngeal carcinoma tissues were highly expressed as shown by the immunohistochemical staining technique. These annexins were tested with immunohistochemical staining but were not found by differential proteomics analysis, and the main reason for this is that proteins of low abundance are not easy to test for due to limited sensitivity. The results displayed reveal that every member may play different roles in cancer cells, and one member may play the major role, but other members minor roles. With regard to breast cancer, annexin A2 was associated with invasion and metastases of cancer cells but annexin A4 and A5 could also contribute to tumorigenesis and the biological behavior of malignant cells. Similarly, annexin A4 and A5 affected cancer cells of pancreatic cancer and laryngeal carcinoma, respectively, while others with low abundance interfere in biological behaviors of malignant cells, but whether the specific mechanism that caused the origin and development of malignant tumors is synergistically affected by annexins is unclear.
Proliferation and invasion of breast cancer cells had decreased when annexin A2 was intercepted by RNA interference (RNAi) (21). We also confirmed that annexin A2 was overexpressed in human breast cancer tissues but others could not be observed by differential proteomics analysis. The results showed that annexin A2 may be important in the invasion and metastasis of breast cancer cells. Although certain proteins are expressed in low abundance, we do not neglect the effects these may have on the biological activities of cells. Overexpression of annexin A4 and A5, but low annexin A1 expression, was observed by immunohistochemical staining, which showed that they functioned in cancer cells with much lower abundance. Annexin A1, which was expressed at a low level in the majority of breast carcinoma samples, was thought to enhance apoptosis (1), and result in cancer cell development and progression. Annexin A4 belongs to the annexin family, but its functions remain unclear. Overexpression of annexin A4 was observed in SCM-1 cells with H. pylori infection, which indicated that annexin A4 may be a potential novel molecular marker for gastric cancer (2). Annexin A5 is another annexin that may be involved in apoptosis, higher tumor stage and poor prognosis, therefore it may contribute to the migration and invasion of cancer cells (20). The results showed that development of breast cancer was a complex biological process, and annexin A1, A2, A4 and A5 synergistically play important roles in apoptosis, carcinogenesis, migration and invasion of cancer cells. Similarly, annexin A2, A4 and A5 were highly expressed in laryngeal carcinoma, which indicated that they may also contribute their respective functions to the origin and development of laryngeal carcinoma. However, annexin A1 was overexpressed in laryngeal carcinoma cells, which was different from breast cancer, and this may result from the difference between laryngeal carcinoma and breast cancer. Annexin A1 is a soluble cytoplasmic protein, moving to membranes when calcium levels are elevated. Annexin A1 was downregulated in dysplastic, tumorous and metastatic lesions, and it may progressively migrate from the nucleus towards the membrane during laryngeal tumorigenesis (22).

Annexin A4 was reported to be upregulated in pancreatic intraepithelial neoplasia cells and may be involved in pancreatic tumor progression (23). The annexin A group mainly exists in humans, and annexin B in animals. We found that upregulation of annexin A4 was observed in golden hamster pancreatic cancer tissues but not in humans. The results showed that annexin A4 could play different roles in golden hamster pancreatic cancer than in humans.

On the whole, malignant tumors are complex diseases, which have many factors involved. Among the annexin A group, annexin A1, A2, A4 and A5 play important roles in breast cancer, pancreatic cancer and laryngeal carcinoma, alone and/or synergistically, by regulating apoptosis, carcinogenesis, migration and invasion of cancer cells, and they may be targets of therapy for malignant tumors. The choice of which annexins to target should depend on their biological behaviors.

\section{Acknowledgements}

The study was supported by funds from the Natural Science Foundation of China (81172496) and the Science and Technology Prop up Support Project of Sichuan (2009SZ0116). 


\section{References}

1. Chuthapisith S, Bean BE, Cowley G, et al: Annexins in human breast cancer: Possible predictors of pathological response to neoadjuvant chemotherapy. Eur J Cancer 45: 1274-1281, 2009.

2. Lin LL, Chen CN, Lin WC, et al: Annexin A4: a novel molecular marker for gastric cancer with Helicobacter pylori infection using proteomics approach. Proteomics Clin Appl 2: 619-634, 2008.

3. Qi YJ, Wang LD, Jiao XY, et al: Dysregulation of annexin II expression in esophageal squamous cell cancer and adjacent tissues from a high-incidence area for esophageal cancer in Henan province. Ai Zheng 26: 730-736, 2007 (In Chinese).

4. Ji NY, Park MY, Kang YH, et al: Evaluation of annexin II as a potential serum marker for hepatocellular carcinoma using a developed sandwich ELISA method. Int J Mol Med 24: 765-771, 2009.

5. Rodrigo JP, Garcia-Pedrero JM, Fernandez MP, Morgan RO, Suárez C and Herrero A: Annexin A1 expression in nasopharyngeal carcinoma correlates with squamous differentiation. Am J Rhinol 9: 483-587, 2005.

6. Kim JK, Kim PJ, Jung KH, et al: Decreased expression of annexin A10 in gastric cancer and its overexpression in tumor cell growth suppression. Oncol Rep 24: 607-612, 2010.

7. Gerke V and Moss SE: Annexins: from structure to function. Physiol Rev 82: 331-371, 2002.

8. Deng S, Zhou H, Xiong R, et al: Over-expression of genes and proteins of ubiquitin specific peptidases (USPs) and proteasome subunits (PSs) in breast cancer tissue observed by the methods of RFDD-PCR and proteomics. Breast Cancer Res Treat 104: 21-30, 2007.

9. Marzook H, Li DQ, Nair VS, et al: Metastasis-associated protein 1 drives tumor cell migration and invasion through transcriptional repression of RING finger protein 144A. J Biol Chem 287 5615-5626, 2012.

10. Singh RD, Haridas N, Patel JB, Shah FD, Shukla SN, Shah PM and Patel PS: Matrix metalloproteinases and their inhibitors: correlation with invasion and metastasis in oral cancer. Indian J Clin Biochem 25: 250-259, 2010.

11. Rohwer N, Lobitz S, Daskalow K, et al: HIF-1alpha determines the metastatic potential of gastric cancer cells. Br J Cancer 100: 772-781, 2009 .
12. Yi S, Guangqi $\mathrm{H}$ and Guoli $\mathrm{H}$ : The association of the expression of MTA1, nm23H1 with the invasion, metastasis of ovarian carcinoma. Chin Med Sci J 18: 87-92, 2003.

13. Wang KL, Wu TT, Resetkova E, et al: Expression of annexin A1 in esophageal and esophagogastric junction adenocarcinomas: association with poor outcome. Clin Cancer Res 12: 4598-4604, 2006.

14. Sharma M, Ownbey RT and Sharma MC: Breast cancer cell surface annexin II induces cell migration and neoangiogenesis via tPA dependent plasmin generation. Exp Mol Pathol 88: 278-286, 2010.

15. Inokuchi J, Narula N, Yee DS, Skarecky DW, Lau A, Ornstein DK and Tyson DR: Annexin A2 positively contributes to the malignant phenotype and secretion of IL-6 in DU145 prostate cancer cells. Int J Cancer 124: 68-74, 2009.

16. Moon HS, Lee YS and Chung HW: The annexin II expression in invasive cervical cancer. Korean J Gynecol Oncol 18: 8-16, 2007.

17. Huang Y, Jin Y, Yan CH, et al: Involvement of annexin A2 in p53 induced apoptosis in lung cancer. Mol Cell Biochem 309: $117-123,2008$.

18. Chan CM, Wong SC, Lam MY, et al: Proteomic comparison of nasopharyngeal cancer cell lines C666-1 and NP69 identifies down-regulation of annexin II and beta2-tubulin for nasopharyngeal carcinoma. Arch Pathol Lab Med 2132: 675-683, 2008.

19. Duncan R, Carpenter B, Main LC, Telfer C and Murray GI: Characterisation and protein expression profiling of annexins in colorectal cancer. Br J Cancer 98: 426-433, 2008.

20. Xue G, Hao LQ, Ding FX, et al: Expression of annexin A5 is associated with higher tumor stage and poor prognosis in colorectal adenocarcinomas. J Clin Gastroenterol 43: 831-837, 2009.

21. Zhang J, Guo B, Zhang Y, Cao J and Chen T: Silencing of the annexin II gene down-regulates the levels of S100A10, c-Myc, and plasmin and inhibits breast cancer cell proliferation and invasion. Saudi Med J 31: 374-381, 2010.

22. Alves VA, Nonogaki S, Cury PM, et al: Annexin A1 subcellular expression in laryngeal squamous cell carcinoma. Histopathology 53: 715-727, 2008.

23. Sitek B, Sipos B, Alkatout I, et al: Analysis of the pancreatic tumor progression by a quantitative proteomic approach and immunhistochemical validation. J Proteome Res 8: 1647-1656, 2009. 